\title{
Financial derivatives and risk models
}

This issue has collected six papers devoted to innovations in two separate topics, namely the development of risk models and techniques in pricing financial derivatives. The papers have been presented in the International Conference on Applied Statistics and Financial Mathematics (ASFM2010) and International Research Forum on the current global economic crisis held in December 2010 in Hong Kong, China.

In the paper "On a discrete-time risk model with delayed claims and dividends" by Yuen, $\mathrm{Li}$ and $\mathrm{Wu}$, the Gerber-Shiu discounted free function for the compound binomial risk model with by-claims and randomized dividend policy is studied. The explicit expression for the Gerber-Shiu discounted free function is obtained. This result allows us to derive formulae for some useful insurance quantities, including the ruin probability, the density of the deficit at ruin, the joint density of the surplus immediately before ruin and the deficit at ruin, and the density of the claim causing ruin.

In the paper "A class of time inconsistent risk measures and backward stochastic Volterra integral equations" by Wang and Shi, a new class of time inconsistent dynamic coherent risk measures is derived, allowing the interest rate involved to be random. In the procedure, one important mathematical tool is a kind of backward stochastic Volterra integral equations with stochastic Lipschitz coefficients. The existence and uniqueness of solutions have been developed.

In the paper "A mean-variance portfolio selection problem subject to a benchmark constraint: An existence result" by Yam, Yung and Zhou, a meanvariance portfolio selection problem that has a benchmark constraint is studied. This constraint changes the problem into a nonconvex one but could be solved via the method of Lagrange multipliers, whose existence is crucial in the solution.

In the paper "Valuation of credit contingent interest rate swap" by Liang and $\mathrm{Xu}$, a tractable and flexible pricing model for credit contingent interest rate swap (CCIRS) is established, which is sensitive to interest rate and credit risk. Intensity-based approach is adopted to construct models for risk-free interest rate and default intensity. By using multi-factor affine jump diffusion, a semi-closed solution to the price of the CCIRS is derived.

In the paper "Discontinuous piecewise polynomial collocation in two dimensions" by Loustau, Irwin, Lindorff and Svadlenka, a collocation method based on a piecewise polynomial approach in two spatial dimensions is considered for solving the Black-Scholes equation. Pricing for barrier options is carried out and the mean-exit-time equation from a price corridor as an estimator of the practical life of an option is investigated.

In the paper "Fast evaluation of some probability integrals arisen from the valuations of discretely monitored derivative securities" by Fung, Li, Yung and Zhou, a fast numerical method to evaluate discretely monitored derivatives is proposed. The key is to compute the involved probability integrals interactively. The method can avoid the "curse of dimensionality" that most other methods suffer. Explicit error estimations are also obtained.

Cedric Yiu Guest Editor 